\title{
Dimensionamento econômico de profundidades e espaçamentos de drenos para a produção de cana-de-açúcar
}

\author{
Rafael Mingoti', Sergio N. Duarte', Jarbas H. de Miranda ${ }^{2}$ \& Décio E. Cruciani ${ }^{2}$
}

\begin{abstract}
RESUMO
Com o aumento do preço da terra e da demanda por álcool/açúcar tem-se buscado o aumento da produtividade da cultura da cana e o aproveitamento de novas áreas para a produção, as quais podem necessitar de drenagem. Com isso, tornam-se importantes estudos de dimensionamento de sistemas de drenagem agrícola e sua avaliação econômica. O trabalho teve como objetivo avaliar economicamente, com o auxílio do modelo SISDRENA, a melhor combinação entre profundidade e espaçamento de valas, implantados em três tipos de solo, com condutividades hidráulicas de 1,0, 0,5 e $0,1 \mathrm{~m} \mathrm{~d}^{-1}$. As profundidades simuladas variaram de 0,20 a 2,00 m, com intervalos de 0,05 m, enquanto os espaçamentos oscilaram de 5 a $100 \mathrm{~m}$, com intervalos de $5 \mathrm{~m}$. Para os três tipos de solo obtiveram-se combinações que revelaram viabilidade econômica para implantação dos sistemas de drenagem. Os resultados indicaram que fatores como o déficit hídrico, a perda de área cultivada pela abertura de valetas e o retorno econômico do empreendimento, são aspectos importantes e devem ser considerados no dimensionamento do sistema de drenagem.
\end{abstract}

Palavras-chave: drenagem, modelagem, lençol freático

\section{Economic design of drain depths and spacings for sugar cane production}

\begin{abstract}
With the increase of the agricultural land prices and the demand for alchol/sugar, there have been a tendency to increase productivity and use of new areas for crop production in which drainage may be needed. Therefore, the studies of economic evaluation of agricultural drainage projects for this crop have become important. This work had as objective to evaluate economically the best combination between depth and spacing of open drains, implanted in three soil types, with hydraulic conductivities of 1.0, 0.5 and $0.1 \mathrm{~m} \mathrm{~d}^{-1}$, with the support of SISDRENA model. The simulated depths varied from 0.20 to $2.0 \mathrm{~m}$, with intervals of $0.05 \mathrm{~m}$, while the spacings varied from 5 to $100 \mathrm{~m}$, with intervals of $5 \mathrm{~m}$. Combinations for the three soils types showed economic viability for the implementation of drainage system. The results also showed that factors such as the water deficit, the cultivated area lost by the opening of drains and the economical return of investment are important aspects and should be considered in the drainage system design.
\end{abstract}

Key words: drainage, modeling, water table

${ }^{1}$ Aluno de graduação em Engenharia Agronômica ESALQ-USP, Av. Pádua Dias, 11 CP 09, CEP: 13418-900, Piracicaba, SP. Fone: (19) 3429-4217, ramal: 264 E-mail: ramingoti@uol.com.br

2 ESALQ/USP, Av. Pádua Dias 11, CP 09, CEP 13418-900, Piracicaba, SP. Fone: (19) 3429-4217. E-mail: snduarte@esalq.usp.br; jhmirand@esalq.usp.br; cruciani@esalq.usp.br 


\section{INTRODUÇÃO}

No rico panorama apresentado pela agricultura brasileira no início do século XXI, destacam-se agronegócios que movimentam expressivas somas a partir de amplas e complexas cadeias produtivas; dentre esses agronegócios, o da canade-açúcar assume posição de destaque, por sua versátil e estratégica funcionalidade de ser matéria-prima, tanto para a produção do açúcar quanto para o álcool, alimento e energia, fontes de divisas de primeira grandeza, além da grande importância social pela mão-de-obra empregada (Formaggio, 2004). Dentre os Estados que apresentam as maiores produções de cana estão, em ordem de classificação, São Paulo, Paraná e Alagoas (Duarte et al., 2001). A maior parte da cana produzida no Estado de São Paulo é cultivada em condições de sequeiro e o aproveitamento de novas áreas para a sua produção, nas quais possa haver necessidade de drenagem, torna-se imprescindível, desde que se obedeça a legislação ambiental vigente; assim, a avaliação econômica para a implantação de novos projetos de drenagem é de fato uma estratégia relevante.

O SISDRENA - Sistema de Drenagem (Miranda et al., 1998), é um modelo de avaliação de desempenho de sistemas de drenagem filosoficamente semelhante ao modelo norte-americano Drainmod (Skaggs, 1990). Trata-se de um modelo unidimensional, que contabiliza os principais componentes do balanço diário de água, em um volume de solo homogêneo e de superfície unitária, localizado eqüidistante entre dois drenos paralelos, e que se estende desde uma eventual camada de impedimento existente no subsolo até a superfície do solo.

O advento do uso de modelos de simulação de desempenho de sistemas de drenagem, gerou grande quantidade de trabalhos que procuram calcular o espaçamento mais econômico entre drenos fechados e abertos (Duarte et al., 1998; Miranda et al., 1998; Borges Júnior et al., 2001); entretanto, pouco se tem feito para se avaliar o efeito da profundidade de instalação dos drenos. A profundidade dos drenos está diretamente relacionada ao espaçamento, pois depende da melhor relação encontrada entre o custo de um metro de dreno instalado e o número de drenos implantados na área (Duarte et al., 2001); é também influenciada pela profundidade ótima de operação da máquina que vai proceder à instalação, pela presença de camadas impermeáveis no subsolo e por limitações quanto à cota de saída por gravidade existente (Ferreira, 1987).

No Estado de São Paulo, as empresas que implantam sistemas de drenagem para o cultivo da cana-de-açúcar costumam utilizar uma profundidade padrão de $1,40 \mathrm{~m}$ para valetas, independentemente das propriedades físico-hídricas do solo, com espaçamentos de drenos variando de 80 a $100 \mathrm{~m}$. Duarte et al. (2001) adaptaram o Sisdrena para realizar simulações específicas para a cultura da cana-de-açúcar cultivada em solo com presença de drenos abertos, objetivando avaliar o melhor espaçamento para drenos implantados a uma profundidade constante de 1,40 m e obtiveram espaçamentos menores que os que vêm sendo utilizados na prática. Este trabalho foi realizado com o objetivo de avaliar a melhor combinação econômica entre diferentes profundidades e espaçamentos de drenos abertos para a produção da cana-deaçúcar, usando o referido modelo.

\section{MATERIAL E MÉTODOS}

Foram realizadas simulações utilizando-se 85 anos de dados diários de chuva e temperatura do ar (1917 a 2001) de Piracicaba, SP (Lat. $22^{\circ} 42^{\prime}$ S, Long. $47^{\circ} 38^{\prime} \mathrm{W}$ e altitude de $546 \mathrm{~m}$ ). A evapotranspiração potencial foi determinada pelo método de Thornthwaite (1948) e Pereira (1997). Foram considerados três perfis de solo homogêneos, cujas características físico-hídricas estão resumidas na Tabela 1.

Tabela 1. Parâmetros físico-hídricos dos três tipos de solo utilizados na simulação

\begin{tabular}{|c|c|c|c|c|c|}
\hline \multirow{2}{*}{ Tipo de Solo } & $\theta_{\text {SAT }}$ & $\theta_{c c}$ & $\theta_{\mathrm{PMP}}$ & $\theta_{\mathrm{SAT}}-\boldsymbol{\theta}_{\mathrm{PMM}}$ & \multirow{2}{*}{ Textura } \\
\hline & & & $\left.n^{3} m^{-3}\right)$ & & \\
\hline $\begin{array}{c}\text { Solo } 1 \\
\left(\mathrm{~K}_{0}=1,0 \mathrm{~m} \mathrm{~d}^{-1}\right)\end{array}$ & 0,572 & 0,434 & 0,189 & 0,245 & Franco-Argilosa \\
\hline $\begin{array}{c}\text { Solo } 2 \\
\left(\mathrm{~K}_{0}=0,5 \mathrm{~m} \mathrm{~d}^{-1}\right)\end{array}$ & 0,645 & 0,577 & 0,444 & 0,133 & Argilosa \\
\hline $\begin{array}{c}\text { Solo } 3 \\
\left(\mathrm{~K}_{0}=0,1 \mathrm{~m} \mathrm{~d}^{-1}\right)\end{array}$ & 0,494 & 0,443 & 0,329 & 0,114 & Franco-Arg.-Siltosa \\
\hline
\end{tabular}

Por operar com dados diários, o Sisdrena estima o escoamento superficial pelo método do Curve Number (CN) (SCS, 1972) e, especificamente, para os solos utilizados, os valores de $\mathrm{CN}$ adotados foram 64, 85 e 90, para os solos 1, 2 e 3 , respectivamente. As profundidades dos drenos simuladas variaram de 0,20 a 2,00 m, com intervalos de 0,05 m.

Por sua vez, os espaçamentos simulados entre drenos variaram de 5 a $100 \mathrm{~m}$, com intervalos de $5 \mathrm{~m}$. Considerou-se que a cultura de cana-de-açúcar, com ciclo de um ano, seria plantada em julho do ano corrente e colhida em junho do ano seguinte e possuiria três estádios de desenvolvimento, conforme estabeleceu Scardua (1985): estabelecimento vegetativo (125 dias), formação da produção (120 dias) e maturação (120 dias).

O sistema de drenagem proposto consistiu no uso de drenos abertos paralelos (valetas). Os parâmetros geométricos do sistema, necessários à simulação, são apresentados na Tabela 2.

O modelo Sisdrena foi utilizado para determinar os valores do índice $\mathrm{SEW}_{30}$ que representam o somatório das alturas diárias de lençol freático acima de um plano localizado a $0,3 \mathrm{~m}$ abaixo da superfície do solo.

Tabela 2. Geometria dos sistemas de drenagem propostos

\begin{tabular}{lc}
\hline \multicolumn{1}{c}{ Parâmetros } & Valores adotados \\
Largura da base da valeta & $0,4 \mathrm{~m}$ \\
Inclinação dos taludes & $1,25: 1$ (solo1); 1,0:1 (solo2); \\
Profundidade da camada impermeável & $0,75: 1$ (solo3) \\
\hline
\end{tabular}


A produtividade relativa esperada que contabiliza apenas o efeito do excesso d'água (YRW) foi calculada por uma relação experimental obtida por Carter et al. (1985) que é função do $\mathrm{SEW}_{30}$. Para contabilizar o efeito da deficiência hídrica sobre a produtividade da cana-de-açúcar (YRD) utilizou-se uma função de produção proposta por Scardua (1985) que é função do déficit de evapotranspiração (razão entre a evapotranspiração real e evapotranspiração potencial) calculado pelo modelo Sisdrena. A produtividade relativa total (YT, decimal), considerando-se conjuntamente os efeitos do excesso e do déficit de água, foi calculada como o produto de YRW por YRD, tal como proposto por Skaggs (1990).

A metodologia utilizada também contemplou o fato de que, para cada profundidade e espaçamento utilizados, haveria uma área perdida que representaria a área não utilizável, devido à abertura das valetas, mais um carreador de $4 \mathrm{~m}$ ao lado de cada valeta necessário para se realizar sua limpeza e outros tratos culturais; esta área perdida foi considerada nos cálculos de produtividade relativa corrigida (YTC, decimal). A análise econômica foi realizada por meio do cálculo do índice valor presente líquido (VPL), seguindo a metodologia utilizada por Duarte et al. (2001). Os valores utilizados para a análise econômica são apresentados na Tabela 3.

Tabela 3. Valores utilizados na análise econômica

\begin{tabular}{|c|c|}
\hline Item & Valor utilizado \\
\hline Produção potencial máxima para a cana-de-açúcar & 200 t ha-1 $^{-1}$ \\
\hline Preço de venda da cana-de-açúcar (*) & $\mathrm{R} \$ 20,06 \mathrm{t}^{-1}$ \\
\hline Custo de produção da cultura (*) & $R \$ 17,11 t^{-1}$ \\
\hline Custos de saneamento e abertura dos drenos coletores $\left({ }^{* \star}\right)$ & RS 350,00 ha $^{-1}$ \\
\hline Custo da abertura de valetas $\left({ }^{* *}\right)$ & $\mathrm{R} \$ 1,30 \mathrm{~m}^{-3}$ \\
\hline Vida útil do projeto & 25 anos \\
\hline Taxa de juros anual & $12 \%$ a.a \\
\hline Preço da terra valorizada $\left({ }^{* \star \star}\right)$ & $\mathrm{R} \$ 6.094,93 \mathrm{ha}^{-1}$ \\
\hline Preço da terra desvalorizada & $\mathrm{R} \$ 2.518,60 \mathrm{ha}^{-1}$ \\
\hline Preço do arrendamento da terra & $\mathrm{R} \$ 322,91 \mathrm{ha}^{-1}$ ano- $^{-1}$ \\
\hline
\end{tabular}

A receita bruta anual, os custos de implantação do projeto e de manutenção do sistema, cálculo da "receita sucata", que envolve o que o produtor ganha em relação à valorização da terra devido à implantação do sistema de drenagem, e os custos anuais, foram calculados de acordo com a metodologia proposta por Duarte at al. (2001).

Ante esses valores de receitas e custos, determinou-se o índice VPL, mediante a Eq. 1, que define a combinação entre profundidade e espaçamento mais econômica. Valores positivos de VPL indicam lucratividade e negativos indicam prejuízos.

em que:

$$
\mathrm{VPL}=\sum_{\mathrm{i}=1}^{\mathrm{i}=\mathrm{n}}\left(\frac{\mathrm{R}(\mathrm{i})-\mathrm{C}(\mathrm{i})}{(1+\mathrm{r})^{\mathrm{i}}}\right)-\mathrm{CI}+\frac{\mathrm{RF}}{(1+\mathrm{r})^{\mathrm{n}}}
$$

VPL - valor presente líquido anual, $\mathrm{R} \$ \mathrm{ha}^{-1}$

$\mathrm{r}$ - taxa de juros anual, decimal

$\mathrm{R}(\mathrm{i})$ - receita anual; $\mathrm{R} \$ \mathrm{ha}^{-1}$
C(i) - custo anual de manutenção do sistema de drenagem, $\mathrm{R} \$ \mathrm{ha}^{-1}$

$\mathrm{RF}$ - incremento no preço de venda da terra devido à drenagem, valor residual, $\mathrm{R} \$ \mathrm{ha}^{-1}$

CI - custo de implantação do sistema de drenagem, $\mathrm{R} \$$ ha $^{-1}$

$\mathrm{n}$ - número de anos da vida útil do projeto

\section{RESULTADOS E DISCUSSÃO}

As isolinhas de produtividades relativas corrigidas YTC, ou seja, descontando-se a perda de área devido à abertura das valetas, obtidas pelo modelo Sisdrena, para os solos 1,2 e 3, são mostradas na Figura 1.

Como se observa, as combinações de 1,15 e $60 \mathrm{~m} ; 1,95$ e $65 \mathrm{~m}$; 2,0 e $30 \mathrm{~m}$, para os solos 1, 2 e 3, foram as que apresentaram os maiores valores de produtividade relativa corrigida correspondendo, portanto, a 73,38, 61,32 e 50,60\%, respectivamente, indicando, portanto, a importância da condutividade hidráulica do solo nas equações de drenagem que, na combinação espaçamento versus profundidade adequada, permite maiores YTC em solos drenados artificialmente.

As isolinhas de valor presente líquido (VPL), obtidas pelo modelo Sisdrena, para os solos 1,2 e 3, são apresentadas na Figura 2.

Tem-se que as combinações de profundidade e espaçamento que proporcionaram maiores valores de VPL, foram 1,05 e 55 m; 1,05 e $40 \mathrm{~m}$; 1,65 e $30 \mathrm{~m}$, para os solos 1,2 e 3 , respectivamente. Referidas combinações corresponderam aos valores de receita líquida de R\$11.001,52, 7.223,90 e 3.587,49 ha-1, demonstrando que, mesmo com o solo de menor condutividade hidráulica (solo 3, Ko $=0,1 \mathrm{~m} \mathrm{~d}^{-1}$ ) é possível se obter um empreendimento economicamente rentável.

Na Figura 2A observa-se a região correspondente à isolinha de 10 mil reais, na qual ocorre o valor máximo de VPL para o solo 1; acima e à direita desta região, os valores de VPL diminuem, visto que as profundidades dos drenos ficam menores e os espaçamentos maiores, havendo menor retirada de água da área drenada; abaixo e à esquerda desta região, os valores de VPL também diminuem, pois as profundidades ficam maiores e os espaçamentos menores, ocorrendo um efeito conjunto de déficit de umidade, de aumento do custo de implantação e de perda de área cultivada.

Nas Figuras 2B e 2C observa-se que as regiões contidas nas isolinhas correspondentes ao VPL máximo (R \$ 6.000,00 e R $\$ 2.000,00$, respectivamente) encontram-se mais verticalizadas que a isolinha de $\mathrm{R} \$ 10.000,00$ da Figura 2A. Assim, verifica-se que à medida que a condutividade hidráulica diminui existe uma tendência da combinação mais econômica entre espaçamento e profundidade de drenos ocorrer para um espaçamento menor e profundidade maior.

Ainda segundo a Figura 2A (solo 1), a recomendação prática de se utilizar drenos a 1,40 m de profundidade, espaçados $80 \mathrm{~m}$, representa uma alternativa ainda interessante para este tipo de solo, de vez que este ponto se localiza dentro da isolinha de VPL de R\$ 10.000,00; entretanto, para o solo 2 
Espaçamento (m)

A.

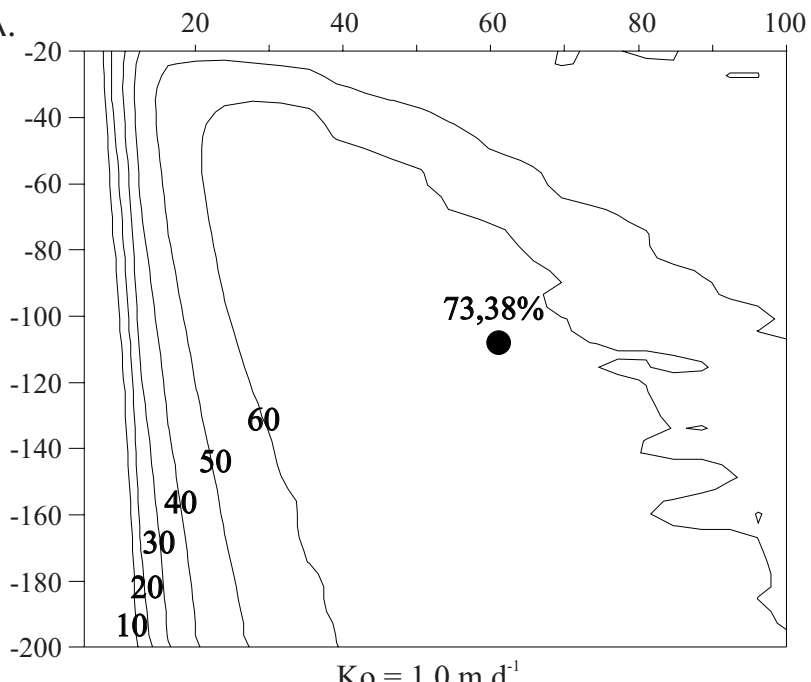

B.

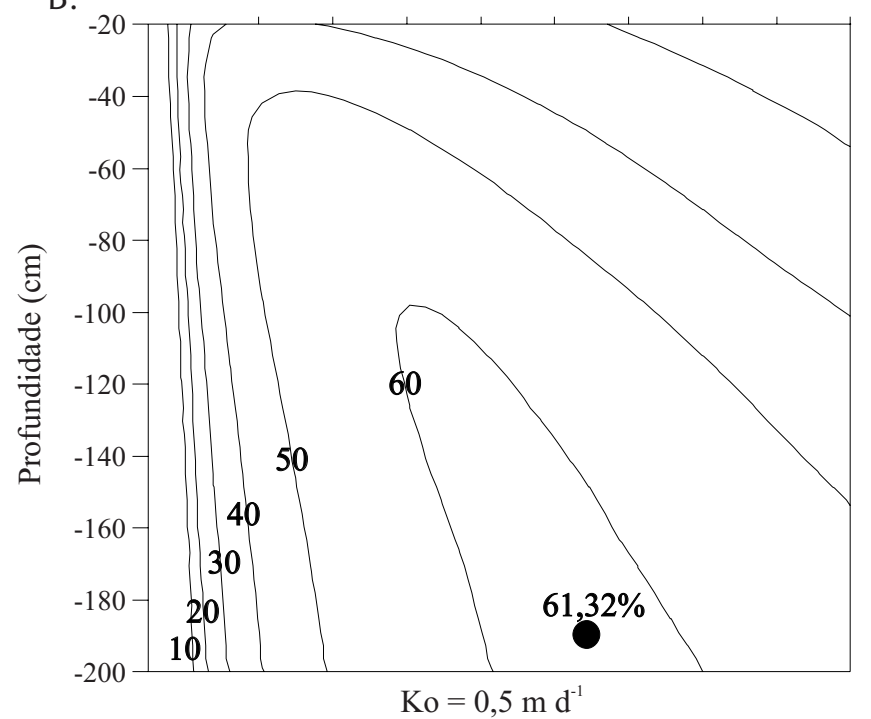

C.

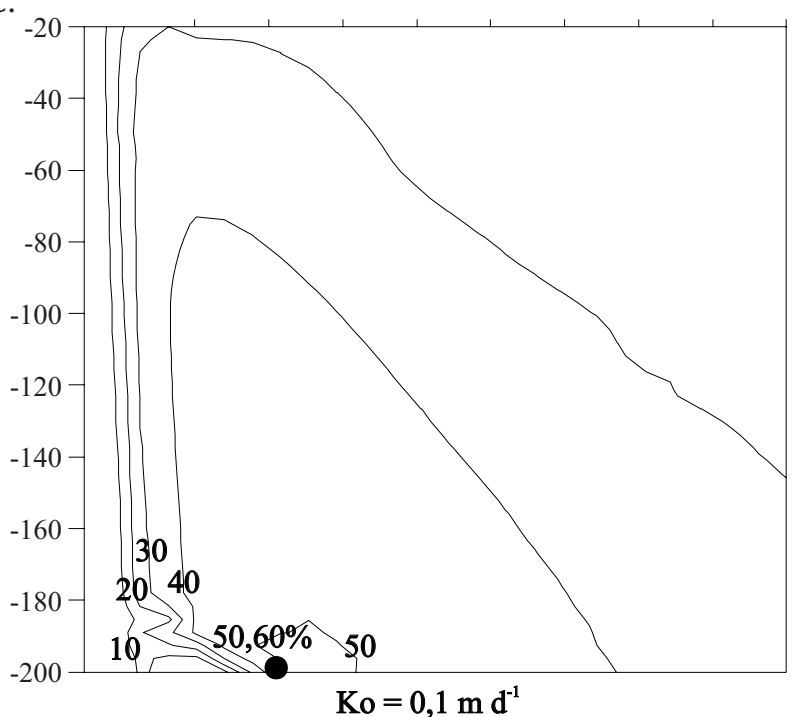

Figura 1. Isolinhas de mesma produtividade relativa corrigida (YTC, \%), resultantes das diferentes combinações entre profundidade e espaçamento de drenos, para os solos 1 (A), 2 (B) e 3 (C)

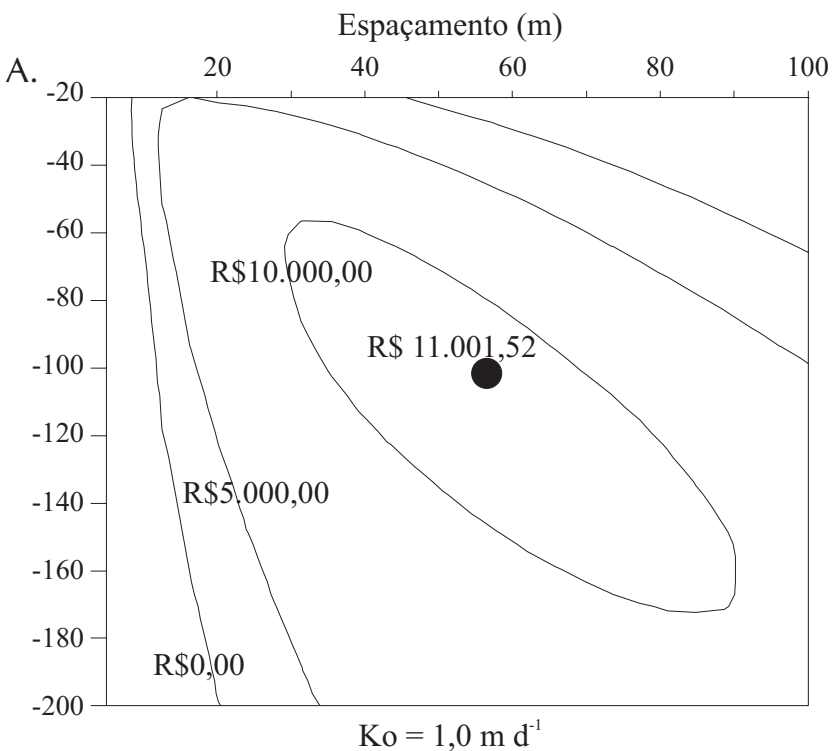

B.

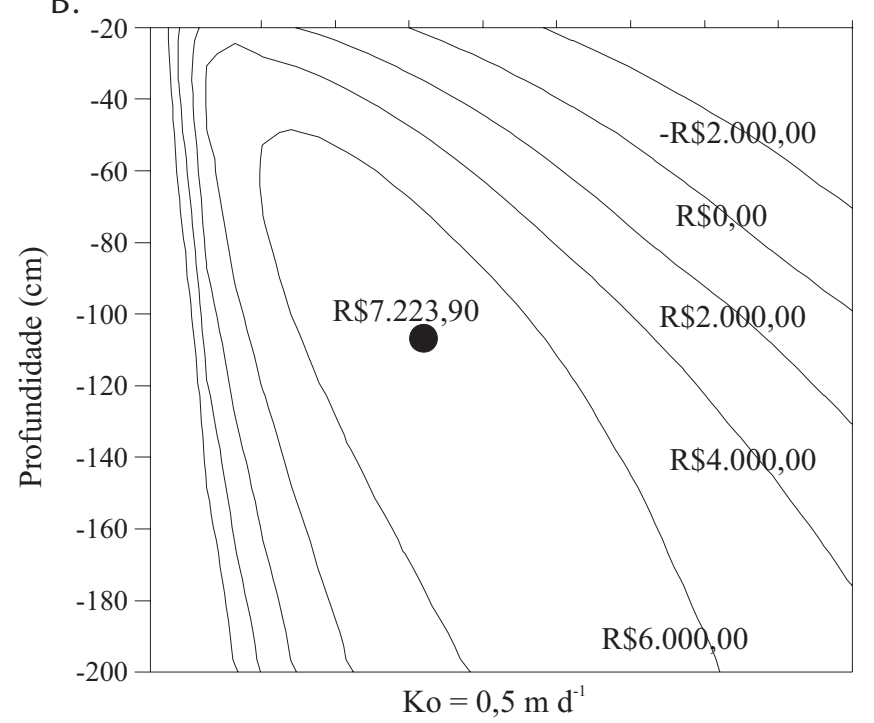

C.

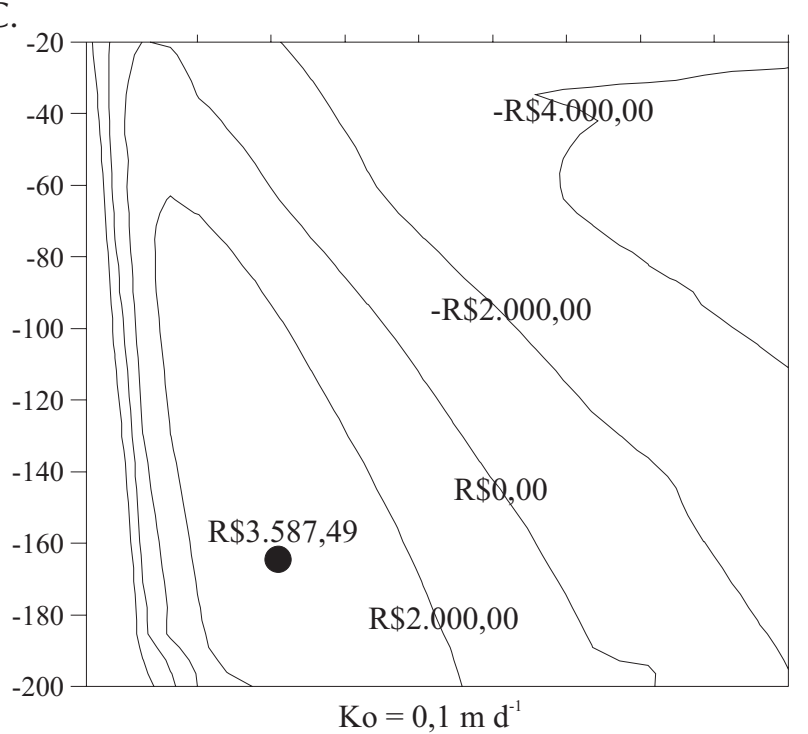

Figura 2. Isolinhas de igual valor presente líquido (VPL), resultantes das diferentes combinações entre profundidade e espaçamento de drenos, para os solos 1 (A), 2 (B) e 3 (C) 
(Figura 2B) esta recomendação traria baixa rentabilidade (VPL menor que R\$ 6.000,00) e, para o solo 3 (Figura 2C) implicaria em prejuízo econômico (VPL de R\$2.000,00).

Os valores de produtividade relativa (YTC) calculados em função da perda de área cultivada para os 85 anos da série estudada, são mostrados na Figura 3. Verifica-se, na referida Figura, uma elevada variação de YTC ao longo da série histórica (mostrada pelos coeficientes de variação superiores a $10 \%$ ), concordando com os resultados encontrados por Rosa (1993). Observando-se a Figura 3A, por exemplo, notase que nove anos (1921, 1928, 1934, 1940, 1958, 1961, 1963, 1975 e 1994) apresentaram valores de YTC consideravelmente abaixo da média.

A análise da causa desses baixos valores de YTC revela que em seis dos nove anos, ocorreu problema de déficit hí-
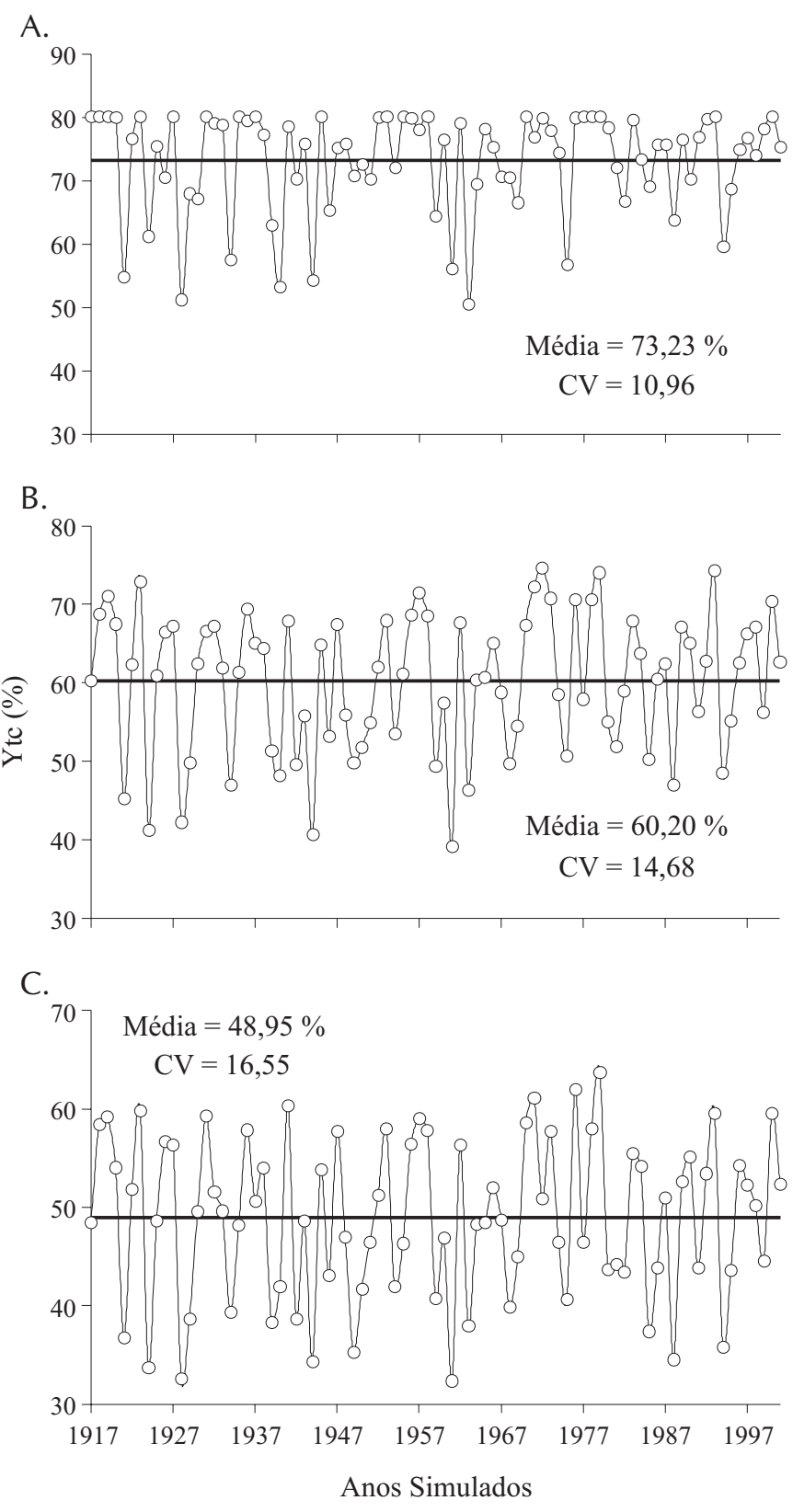

Figura 3. Valores da produtividade relativa corrigida (YTC) variando ao longo dos 85 anos (1917 a 2001) da série de dados climáticos, para os solos 1 (A), 2 (B) e 3 (C) drico sério (YRD menor que 65\%). A investigação das Figuras 3B e 3C conduz a resultados semelhantes, ou seja, como a cultura da cana não é irrigada, não apenas o excesso como também o déficit de umidade, são fatores significativos a serem considerados no dimensionamento do sistema de drenagem, cujos resultados evidenciam a importância da modelagem computacional como ferramenta de dimensionamento e a relevância de se dispor de séries históricas representativas da região.

\section{CONCLUSÕES}

1. As melhores combinações de profundidade e espaçamento de drenos para os solos com condutividade hidráulica de 1,0, 0,5 e 0,1 $\mathrm{m} \mathrm{d}^{-1}$ foram respectivamente, 1,05 e $55 \mathrm{~m}, 1,05$ e $40 \mathrm{~m}$, e 1,65 e $30 \mathrm{~m}$, cujos valores presentes líquidos de R\$11.001,52; 7.223,90 e 3.587,49 por hectare, comprovam a viabilidade econômica desses empreendimentos.

2. O padrão relativamente comum para drenagem de várzeas objetivando-se o cultivo da cana-de-açúcar e se usando valetas de 1,40 m de profundidade, espaçadas cerca de $80 \mathrm{~m}$, reflete uma boa opção econômica apenas para o solo de maior condutividade hidráulica.

3. Os fatores déficit hídrico, perda de área cultivável com a abertura de valetas e retorno econômico do empreendimento, são significativos para a definição da melhor combinação entre profundidade e espaçamento de drenos a ser utilizada.

\section{LITERATURA CITADA}

Borges Júnior, J. C. F.; Ferreira, P. A.; Pruski, F. F.; Silva Junior, A. G. Sistemas de drenagem agrícola. Parte I: Desenvolvimento do modelo e análise de sensibilidade. Revista Brasileira de Engenharia Agrícola e Ambiental, Campina Grande, v.5, p.369375, 2001.

Carter, C. E.; Irvine, J. E.; McDaniel, V.; Dunckelman, J. Yield response of sugarcane to stalk density and subsurface drainage treatments. Transactions of the ASAE, St. Joseph, v.28, n.1, p.172-177, 1985.

Duarte, S. N.; Ferreira, P. A.; Pruski, F. F.; Martinez, M. A. Modelo para avaliação de desempenho de sistemas de drenagem subterrânea e cálculo de espaçamento de drenos. Parte 1: Desenvolvimento e Análise de Sensibilidade. Engenharia Agrícola, Jaboticabal, v.18, n.2, p.19-31, 1998.

Duarte, S. N.; Miranda, J. H.; Folegatti, M. V. Dimensionamento econômico de sistemas de drenagem para a produção de canade-açúcar usando o modelo SISDRENA. Revista Brasileira de Engenharia Agrícola e Ambiental, Campina Grande. v.6, n.1, p.1-6, 2001.

Ferreira, P. A. Drenagem In: Curso de Engenharia de Irrigação. Brasília: ABEAS. 1987. 86p.

Formaggio, A. R. Sensoriamento remoto: Um olhar espacial sobre os canaviais. Visão Agrícola, Piracicaba. v.1, n.1, p.2831, 2004. 
Miranda, J. H.; Duarte, S.N.; Folegatti, M. V. Modelo para simulação da dinâmica da água em sistemas de drenagem subterrânea. Revista de Engenharia Rural, Piracicaba, v.9, n.2, p.1-19, 1998.

Pereira, A. R.; Villa Nova, N. A.; Sediyama, G. C. Evapo(transpi)ração. Piracicaba: FEALQ, 1997, 183p.

Rosa, J. A. Níveis de drenagem para a cultura do feijoeiro em várzeas. Pesquisa Agropecuária Brasileira, Brasília. v.28, n.8, p.947-954, 1993.
Scardua, R. O clima e a irrigação na produção agroindustrial da cana-de-açúcar (Saccharum spp). Piracicaba: ESALQ/USP, 1985. 122p. Tese Livre-Docente

SCS - Soil Conservation Service. National engineering handbook. IV. Hydrology. s.l., USDA, 1972, n.p.

Skaggs, R. W. Drainmod: user's manual. Raleigh: North Carolina State University, 1990. 101p.

Thornthwaite, C. W. An approach toward a rational classification of climate. Geography Review v.38, p.55-94, 1948. 